\title{
Endoscopic Robotically Assisted Coronary Artery Bypass Anastomosis on the Beating Heart: Analysis of Quality and Patency
}

\author{
Marco A. Zenati, M.D., Gilbert J. Burckart, Pharm.D., Bartley P. Griffith, M.D. \\ Division of Cardiothoracic Surgery, University of Pittsburgh Medical Center, \\ Pittsburgh, Pennsylvania, U.S.A. \\ zenatim@msx.upmc.edu
}

\begin{abstract}
Background: Construction of a coronary artery bypass anastomosis on the beating heart using a computer-enhanced telemanipulation system is feasible but its quality is unknown. Methods: In nine pigs the Left Internal Mammary Artery (LIMA) to the Left Anterior Descending (LAD) coronary artery anastomosis was constructed exclusively using the ZEUS System. Pressure-fixation stabilizer provided local immobilization. LIMA flow was analyzed using transit-time technology. Pigs had to survive without ischemia with proximal LAD ligated for one hour. Angiography was performed within 90 minutes of the anastomosis and graded using the Fitzgibbon Classification. After sacrifice, the anastomosis was assessed using probes. Results: LIMALAD anastomosis was successful in all cases in $22 \pm 3.6$ minutes. No repair stitches were necessary. LIMA flow was $21.6 \pm 2.5 \mathrm{ml} / \mathrm{min}$. Angiographic patency was $100 \%$ with grade A anastomosis in all. Eight pigs survived without ischemia for $60 \mathrm{~min}$; one pig fibrillated shortly after completion of anastomosis. A $2 \mathrm{~mm}$ probe was passed without resistance through the anastomosis in all cases. Conclusion: The quality of the LIMA-LAD anastomosis performed on the beating heart using the ZEUS robotic system is excellent. Confirmation of these results with a totally endoscopic approach is warranted.
\end{abstract}

Keywords: Cardiovascular surgery; robotics and robotic manipulators; clinical system development.

\section{Introduction}

The ultimate goal of Minimally Invasive Coronary Surgery is to achieve a totally endoscopic ("ports only") coronary artery bypass operation on the beating heart. The high degree of difficulty associated with the performance of microvascular $(2 \mathrm{~mm})$ endoscopic coronary artery bypass anastomosis on the beating heart without cardiopulmonary bypass, requires dexterity enhancement through robotic technology. Several computer-enhanced telemanipulation surgical systems have been used to test the feasibility of endoscopic beating heart coronary artery bypass graft surgery $(1,2)$. The construction of Left Internal Mammary Artery (LIMA) to Left Anterior Descending (LAD) anastomosis is the critical factor in the operation and current studies have not addressed the quality and patency of endoscopic anastomosis using robotic telemanipulation instruments. We hypothesized that the patency and quality of the LIMA to LAD anastomosis performed exclusively using the ZEUS Surgical 
System (ComputerMotion, Goleta, CA) would be excellent, based on our previous large experience in beating heart coronary bypass surgery and our work with the ZEUS System in cadavers.

\section{Methods}

Nine crossbred swine of either sex weighing an average of $42.5 \mathrm{Kg}$ (range $35-50 \mathrm{Kg}$ ) were used for the study. All animals received humane care in AAALAC, USDA registered facility in compliance with the "Principles of Laboratory Animal Care" formulated by the National Society for Medical Research and the "Guide for the Care and Use of Laboratory Animal Resources" and published by the National Institutes of Health (NIH Publication No. 85-23, revised 1985). The study protocol was approved by the Institutional Animal Care and Use Committee of the University of Pittsburgh (IACUC Approval \#1002). Maintenance of general anesthesia and monitoring was performed as previously described. A median sternotomy was performed and the LIMA was harvested using skeletonization technique. Heparin $(2 \mathrm{mg} / \mathrm{Kg})$ was given intravenously and the LIMA was divided distally and prepared for anastomosis. A pressure-fixation myocardial stabilizer (OPCAB System, Genzyme or CMI-Grace II, ComputerMotion) was used. Three $5 \mathrm{~mm}$ ports were created on the left chest to align instruments with LAD and create a 90' angle between the instruments. The LAD was manually dissected and opened. An intracoronary shunt (Flocoil, Guidant) of appropriate size was introduced in the LAD and distal perfusion was reestablished. From this point on the procedure was performed from the ZEUS surgeon control station, located 10 feet away from the operating table. A PTFE 8-0 double armed suture with TT8 needles was used. After the completion of the anastomosis, the LAD was ligated proximally and flow was measured using transit-time technology (Transonic, Ithaca, NY). Contrast angiography was performed by injection of contrast agent (Renographin, Roche) into the LIMA. Data were expressed as mean \pm standard deviation.

\section{Results}

All 9 LIMA-LAD anastomoses were completed successfully without need for repair stitches and using exclusively robotic telemanipulation in $22 \pm 3.6$ minutes (range 18$30 \mathrm{~min}$ ). The LIMA flow after LAD ligation was $21.6 \pm 2.5 \mathrm{ml} / \mathrm{min}$. Eight animals survived for 60 minutes with the LAD ligated without ischemic EKG changes; one pig suffered from an episode of ventricular fibrillation. Angiographic patency was $100 \%$ with Fitzgibbon Grade A in all. A $2 \mathrm{~mm}$ Parsonnet probe was passed into the anastomosis without resistence after the animal was sacrificed. Upon gross inspection, no evidence of thrombus formation at the anastomotic site was identified.

\section{Discussion}

We demonstrated in a pig beating heart model that an excellent LIMA-LAD anastomosis can be constructed endoscopically using the ZEUS robotic surgical 
system with conventional 2D visualization and 4 degrees of freedom (DOF). Other groups (1) have suggested that a manipulator with less than 7 DOF could not attain general goal positions and orientation in space. We completed all anastomosis in 22 minutes with a 100\% angiographic patency and found the ZEUS System adequate for the task.

\section{References}

5.1. Falk V, Diegler A, Walther T, et al. Endoscopic Coronary Artery Bypass Grafting on the Beating Heart Using a Computer Enhanced Telemanipulation System. Heart Surg Forum 1999;2:199-205

5.2. Reichenspruner $\mathrm{H}$, Boehm DH, Gulbins $\mathrm{H}$, et al. Robotically Assisted Endoscopic Coronary Artery Bypass Procedures without Cardiopulmonary Bypass. J Thorac Cardiovasc Surg 1999;118:960-1.

\section{Acknowledgement}

This work was supported by a Research Grant from the Ravitch/Hirsch Center for Minimally Invasive Surgery of the University of Pittsburgh. 